\title{
Treatment of Post-Herpetic Neuralgia by Prolonged Electric Stimulation
}

\author{
P. W. NATHAN, P. D. WALL
}

British Medical fournal, 1974, 3, 645-647

\section{Summary}

The results of treating patients with severe post-herpetic neuralgia with prolonged self-administered electric stimulation from a portable apparatus were good in 11 out of 30 patients. None of these patients had had as good relief of pain with other forms of treatment. In 10 patients some effects from stimulation continued after stimulation stopped. In eight there was an improvement in the course of the neuralgia, and in two there was a cure.

\section{Introduction}

The starting point for the treatment of severe post-herpetic neuralgia by self-administered transcutaneous electric stimulation was the gate-control theory of pain of Melzack and Wall (1965). An implication of this theory is that exciting the large myelinated cutaneous fibres would reduce the effect of an input via the small myelinated and non-myelinated fibres. As the activity of some of these small fibres causes pain, reducing the effect of their input might diminish the pain. Noordenbos (1959) has argued that the pain and abnormal sensitivity of the skin in this condition are due to a preferential destruction of the larger myelinated afferent nerve fibres, leaving an excessive proportion of small delta fibres and non-myelinated fibres still functioning, and he and Lourie and King (1966) have provided evidence in support of this view. The rationale for using percutaneous electrical stimulation in such a condition is that it would reintroduce the normal inhibition by driving the remaining large fibres to a high level of activity.

Though electrical stimulation for pain has been in the armamentarium of physiotherapists for decades it has now been provided with a rationale by the gate-control theory. Reports on

National Hospital for Nervous Diseases, London WC1N 3BG P. W. NATHAN, M.D., F.R.C.P., M.R.C. External Scientific Staff

Department of Anatomy, University College, London W.C.1 P. D. WALL, M.A., D.M., Professor of Anatomy

its effectiveness in temporarily abolishing chronic pain have been published by Wall and Sweet (1967). On account of this preliminary hopeful report we started in 1969 stimulating the larger rapidly conducting fibres of peripheral nerves in a series of patients with a painful peripheral nerve lesion, including post-herpetic neuralgia. We were hoping that short sessions of stimulation would be followed by lasting relief of pain though we did not think this likely. Our hopes were not fulfilled and our expectations proved correct. As the pain was not felt during low-level electrical stimulation many neurologists and neurosurgeons treating pain thought it reasonable for the patient to stimulate the nerves of the region continuously by wearing a portable stimulator. This continuous stimulation was made easier by the introduction of large electrodes that can be fixed relatively easily to the skin. This is the form of treatment reported here. With this treatment we hoped that there would be relief of pain during the periods of stimulation and we did not expect any lasting or total relief. Our hopes have been fulfilled to a moderate extent and our expectations exceeded.

\section{Patients and Methods}

There were two series of patients with severe post-herpetic neuralgia. The first consisted of nine patients who attended hospital for periods of two to seven years and the second consisted of 21 patients who attended for periods of between one and two years. In both series all forms of therapy had failed, and if any new form were to succeed it would be an advance in the treatment of this most painful condition. In all the patients self-administered prolonged electric stimulation was used only after adequate trials with cold sprays, vibration, and repeated injections of local anaesthetics had failed to help. All patients whose pain was made worse by the light rubbing or brushing of their clothes were fitted with close-fitting polyethylene or Plastozoate jackets if they wanted them.

The nine patients of the first series had passed through three periods of therapy: the first period was before we used any form of electrical stimulation, the second was when we used electrical stimulation intermittently, and the third period is reported here, when the patients treated themselves by transcutaneous stimulation as often and as long as they wanted to. Treatment during the first period consisted of spraying the skin with P.R. Spray (trichlorofluoromethane $85 \%$, dichlorodifluoromethane $15 \%$ ) or 
with ethyl chloride, vibration with a Pifco vibrator, sometimes preceded for several days with treatment with a heavier vibrator, rubbing anaesthetic ointment into the skin if this helped the pain or tenderness, repeated injections of local anaesthetic solutions subcutaneously and into the deeper tissues of the tender region, and similar injections into the supraorbital nerve for postherpetic ophthalmic neuralgia.

Treatment during the second period consisted of bipolar percutaneous stimulation at a frequency of $100 / \mathrm{sec}$, pulse width $0 \cdot 1$. msec; the voltage was controlled by the patient. Various kinds of electrodes were used. They were all small with a maximum diameter of $0.5 \mathrm{~cm}$ and made of steel or of steel covered with lint moistened with water or saline. Frequency of treatments varied between twice a day and twice a week. In most cases the patients had five 15-minute treatment sessions a week.

Patients of both the first and the second series were treated in the third period. In the third period stimulation differed from that of the second period as follows: the patient used the stimulator for as long as he wanted-possibly for 12 hours or more. The apparatus is portable and powered by batteries, and the patient can carry it slung over his shoulder or tucked into a pocket or over a belt; the leads between the stimulator and his skin are usually hidden in his clothing. The electrodes are larger than those used in the second period. These large electrodes help to avoid painful sensations, and with them it is easier to stimulate the nerves transcutaneously and to avoid stimulating the skin itself.

The stimulator we used was either the Stim-Tech stimulator or their more recent model which is pocket-size. This apparatus delivers square-wave pulses with no net direct current component. The voltage output of the stimulator is variable and under the patient's control. The pulse duration is between 50 and 500 usec and frequency between 15 and $180 \mathrm{~Hz}$. The patient can control the voltage, pulse duration, and pulse frequency. The electrodes used were either the $4.5-\mathrm{cm}$ diameter sponge electrodes supplied with the Stim-Tech stimulator or pregelled disposable Gel-Pad electrodes made for E.C.G. recording. The patients were told to test the effects of putting the electrodes in various places in relation to the regions where they had pain and tenderness, to try reversing the polarity with the electrodes in a constant place, and to try altering the various stimulus values. The placement of the electrodes was at first outside the area of skin tenderness, particularly in those patients where the skin was very hyperaesthetic and hyperalgesic. The Stim-Tech stimulator with the electrodes stuck on the skin is shown on a patient with postherpetic neuralgia in the photograph.

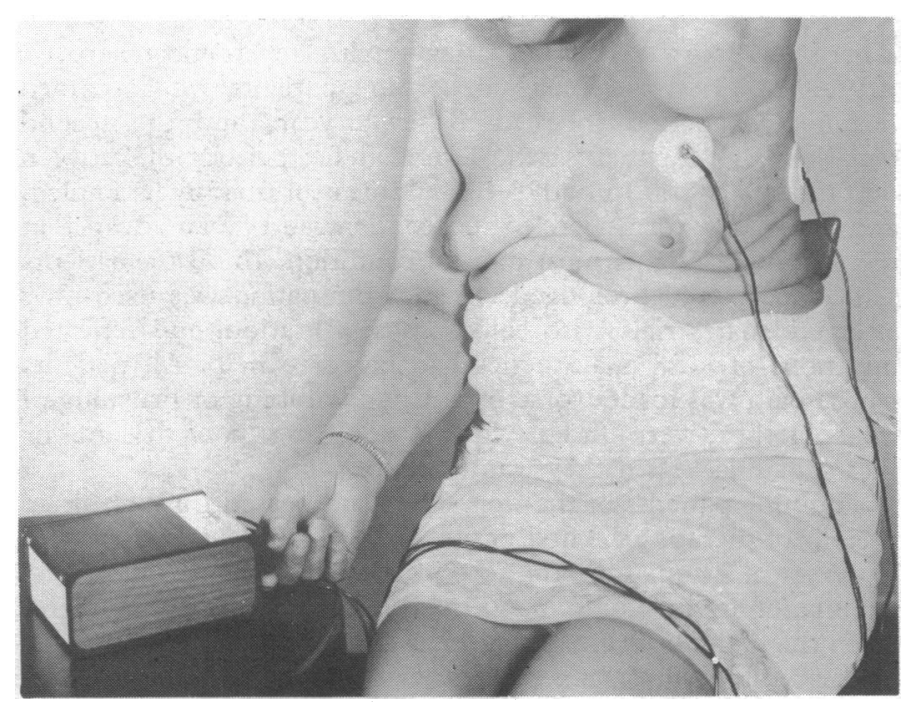

Patient with post-herpetic neuralgia adjusting controls of stimulator on table. Electrodes are stuck to skin, bridging painful region of scarring.
The method of treatment is not free from trouble for the doctor. It is essential to see the patient to notice whether the skin is becoming damaged and also to remind him that the idea is not to cure the pain but alleviate it. This idea seems to be so strange to many patients that though they are often told this they complain that they are not getting any better.

Stimulation as the patients use it causes a tingling sensation free from pain. It is a tactile paraesthesia. When relief is maximal the patient feels this paraesthesia and not his pain, and during stimulation he loses the hyperaesthesia.

\section{Results}

To help us assess the effect of prolonged self-administered electric stimulation the patients were asked to record the periods of stimulation and the state of pain on printed charts. Another way of assessing the effectiveness of the treatment was the reduction in the drugs-analgesics, tranquillizers, antidepressants-that the patient was taking. All patients who received benefit from the treatment spontaneously reduced the amount of medication or allowed us to do so without complaint.

Two of the 30 patients returned their stimulators as they regarded themselves as cured. One had had pain for four years before starting stimulation and he used stimulation for three years. The other had had pain for three years, and he gave up stimulating after four months. Both of these patients have remained untroubled for one year so far. Of the remaining 28 patients 19 returned their stimulators and nine kept them. Of the 19 who returned them four said that stimulation made their pain worse and six said it had no effect. In seven patients pain was relieved during stimulation-only slightly in two cases-but became worse afterwards, and two patients discovered that rest relieved their pain. The 10 patients who found stimulation made the pain worse or who, with experience, found that it had no effect on the pain were the patients with the most painful post-herpetic neuralgia. The two patients who found that resting and relaxation were just as important as the stimulation had stimulators for many months. They undoubtedly got relief from stimulation, and their total state improved. It was when their pain was less obnoxious that they concluded that resting and consciously relaxing were more important than the stimulation. Two other patients thought that the resting during stimulation was an important factor in pain relief though they still thought stimulation was more useful. The observations of these patients brought to our attention this relation between activity and rest and the pain. On inquiry we found that several patients knew that pain came on when they got up in the mornings and started the activities of the day and diminished when they were completely at rest.

Some patients found that this stimulation stopped the pain in some regions and had no effect in others. The region where it was stopped was the region where pain was less severe. Even this may be a great help to the patient for it may remove the pain in a large area and reduce it to a small area.

Altogether 16 patients got some relief during stimulation-six got total relief, seven moderate relief, and three slight relief. In 10 patients relief outlasted stimulation-for more than two hours in seven cases, for one to two hours in two, and for less than one hour in one. Not all the six patients who got complete relief continued stimulating, and two of them found that the pain was worse after they had stopped. It was surprising to find that some patients got relief from pain for as long as four hours after they had stopped stimulating.

It was even more surprising to find that the neuralgia gradually improved in eight patients. No improvement in the neuralgia had occurred in any of the patients before they started prolonged electrical stimulation, and some had been observed for periods of up to seven years.

Of the 22 patients who had stimulators for more than one month four found that the effect of stimulation lessened over the months. These included the two who in the end decided 
that rest was the important factor, but in the first two or three months of their treatment it was obvious that stimulation was more important in relieving pain than rest was.

It seems that patients with the most severe pain do not get relief from electrical stimulation. We have seen six patients (not included in this series) who found straight away that the electric stimulation merely added to their pain. Other patients felt the tingling paraesthesiae and their pain but there was no interaction between the two. Those who got relief from stimulation did not feel the pain while they felt the paraesthesiae or else they felt less pain. It may be that in those patients in whom electrical stimulation increased the pain there were insufficient large myelinated fibres surviving to produce the inhibition and when any fibres were stimulated it was the small myelinated fibres.

Results with continuous electric stimulation were compared with those with cold spray, vibration, repeated injections of the tender area with local anaesthetic solution, and rubbing the skin or tender area. No relation between all these ways of alleviating the pain was found.

An examination of the cases in which stimulation was stopped showed that there were two groups of patients who do not want this treatment: those with the most severe pain in whom stimulation either had no effect or it made the pain worse and those with the least severe pain for whom the whole inconvenience of constant stimulation did not justify their efforts.

\section{Discussion}

Our success with temporary treatment of certain patients with severe post-herpetic neuralgia does not mean that electric stimulation is better than sprays or vibration. The patients chosen for testing electric stimulation were those in which these other forms of stimulation had continually failed. There are cases in which either or both work, and some patients who had electric stimulation considered that vibration or cold sprays were better.

The method has certain disadvantages. The apparatus is expensive, and it is a nuisance to have to carry it around and to wear electrodes attached to the skin. Some old people have refused to take the apparatus as they are frightened of electricity. Patients tend to be disappointed for they expect a cure and are given alleviation.

The mechanism that underlies prolonged relief of pain for hours after stopping stimulation is not understood. From studies of neuromas induced in the rat Wall and Gutnick (1974) have proposed that electric stimulation may silence abnormal generators of nerve impulses in the peripheral nerve itself. They found that a tetanus delivered to the nerve for seconds stopped spontaneous firing for six minutes. The mechanism of the improvement in the whole course of the post-herpetic neuralgia is even more of a puzzle. Possibly prolonged stimulation induces changes in the peripheral nerve itself or it may be that central structures, relieved of a continuous afferent barrage, may sink back to normal excitabilitv.

We thank Dr. J. A. V. Bates, who helped us with servicing the s:imulators, and physicians at the National Hospital for refenring patients to us. This work was supponted by the Medical Research Council.

\section{References}

Lourie, H., and King, R. B. (1966). Archives of Neurology, 14, 313.

Melzack, R., and Wall, P. D. (1965). Science, 150, 971.

Noordenbos, W. (1959). Pain. Amsterdam, Elsevier.

Wall, P. D., and Sweet, W. H. (1967). Science, 155, 108.

Wall, P. D., and Gutnick, M. (1974). Nature, 248, 740.

\title{
Vitamin D and Myocardial Infarction
}

\author{
VICTOR LINDÉN
}

British Medical Fournal, 1974, 3, 647-650

\begin{abstract}
Summary
A detailed investigation was carried out into the consumption of vitamin $D$ from different sources in patients who had suffered from myocardial infarction, angina pectoris, and degenerative joint diseases. Randomly selected controls of the same ages and sex were drawn from the Central Bureau of Statistics. The consumption was significantly higher in infarction patients. A daily intake of $30 \mu \mathrm{g}$ may be the critical level. Student's $t$ test for trend showed increasing probability of myocardial infarction with increasing intake of vitamin $D$, and more infarction patients than controls had a history of kidney stone. Long-term high consumption of vitamin $D$ may be a precipitating cause of myocardial infarction.
\end{abstract}

\section{Introduction}

It is well known that vitamin D produces serious toxic effects if

Institute for Community Medicine, University of Tromso, Norway VICTOR LINDÉN, M.D., Professor consumed for a period in grossly excessive amounts (Lowe et al., 1954; Fellers and Schwartz, 1958; Smith et al., 1959; Lowe, 1965; Kaserer et al., 1966; Seelig, 1969). On the basis of such data The Committee on Nutrition of the American Academy of Pediatrics (1963) recommended the discontinuation of the practice of enriching food other than milk and infant food. The long-term consequences of consumption of more than the recommended allowance of $10 \mu \mathrm{g}$ a day in adults are entirely unknown.

Dalderup et al. (1965), Feenstra and Wilkens (1965), and Palmisano (1973) showed that the consumption of cod liver oil and vitamin D preparations raises serum cholesterol. Cholesterol is probably converted into 7-dehydrocholesterol (vitamin $\mathrm{D}_{3}$ ) in vivo (Glover et al., 1952). Dalderup et al. (1965) and Knox (1973) suggested an association between vitamin D consumption and death from ischemic heart disease. Patients with hypercalcaemia because of vitamin $\mathrm{D}$ intoxication are likely to develop renal calculi. Lindén (1972) and Westlund (1973) found an association between urolithiasis and coronary heart disease. Kinley and Krause (1959) found that vitamin A may lower serum cholesterol levels, and Ross and Campbell (1961) suggested that a relative vitamin A deficiency may cause coronary heart disease. Very little is, however, known of the interrelationship between vitamin $A$ and $D$ in vivo. 